\title{
Systemic autoinflammatory disease and genetic testing
}

Qingping Yao*

Division of Rheumatology, Allergy and Immunology, Department of Medicine, Stony Brook University Renaissance School of Medicine,

Stony Brook, New York 11794, USA

Systemic autoinflammatory disease (SAID) is a new category of inflammatory disorders that are mainly associated with abnormal innate immune responses. These disorders largely include hereditary periodic fever syndromes, Nucleotide binding oligomerization domain containing protein 2 (NOD2)-associated diseases, newly identified monogenic, and known polygenic disorders. ${ }^{[1,2]}$ Unlike typical autoimmune diseases, SAIDs are usually not associated with detectable autoantibodies or specific antigen-stimulated $\mathrm{T}$ cells. Besides relying on clinical phenotypes, a diagnosis of SAID also requires genetic testing in general, particularly for monogenic disorders.

In the area of genomic medicine, genetic testing and molecular analysis are important for the diagnosis of these disorders. Genomic medicine is an emerging medical discipline that involves using genomic information about an individual as part of the clinical care and health outcomes/policy implications of that clinical use..$^{[3]}$ Genetic testing may encompass targeted DNA sequencing (Sanger method), next-generation sequencing (NGS), and whole exome sequencing. NGS may be used to design a gene panel of different combinations. ${ }^{[4]}$ For hereditary periodic fever syndromes, targeted DNA sequencing, for example MEFV mutations for familial Mediterranean fever (FMF), is often ordered if clinically indicated. However, clinical phenotypes of autoinflammatory diseases usually overlap. As a result, a periodic fever syndrome gene panel is preferably ordered. To perform genetic panel testing, NGS is commonly used. For further genetic testing for complex cases of unclear cause or for research purposes, whole exome sequencing may be used..$^{[5]}$

Monogenic hereditary periodic fever syndromes typically include FMF, cryopyrin-associated periodic syndrome (CAPS), hyper IgD syndrome/mevalonate kinase deficiency (MVK), and TNF receptor-associated periodic syndrome (TRAPS), and these disorders are inherited in a Mendelian fashion, i.e., either autosomal recessive or dominant. ${ }^{[6]}$ Generally, the genetic mutations associated with these disorders are highly penetrant with an allele frequency of $<1 \%$ in the general population. However, low-penetrance variants with an allele frequency of $>5 \%$ may be identified and may represent a significant clinical challenge for disease diagnosis. In FMF and CAPS, lowpenetrance genetic variants are detected and clinically used for the diagnosis of these disorders in the presence of characteristic clinical phenotypes, though there has been some controversy. For example, the allele frequency of the MEFV $\mathrm{E} 148 \mathrm{Q}$ is $4.7 \%$ in the healthy Caucasians ${ }^{[7]}$ and up to $23 \%$ in the Japanese population. ${ }^{[8]}$ This variant is used to support the diagnosis of FMF if a typical clinical phenotype is present. The allele frequency of the NLRP3 Q703K is $5-6.5 \%$ in healthy Caucasians. ${ }^{[9-11]}$ This variant may be used to support a diagnosis of CAPS in the presence of a proper clinical phenotype. In addition, in NLRP12-induced autoinflammatory disease, the 
most common genotype reported is the NLRP12 F402, and its allele frequency is $5 \%$ in healthy Caucasians. ${ }^{[12,13]}$

Technicians or researchers who work in molecular laboratories usually report genetic testing results based upon the Joint Consensus Recommendation of the American College of Medical Genetics and Genomics and the Association for Molecular Pathology. The terms "mutation" and "polymorphism" have been currently replaced by the term "variant" with the following modifiers: pathogenic, likely pathogenic, uncertain significance, likely benign, or benign. ${ }^{[14]}$

As far as my experience goes, these molecular laboratories usually do not report the so-called benign genetic variants unless they are requested. Ordering physicians often read the genetic reports and interpret them as such. As a result, some genetic markers such as the low-penetrance variants aforementioned may be missed and proper diagnosis would be delayed. Physicians who specialize in autoinflammatory diseases may be able to pick up the dismissed genetic markers, make a proper diagnosis, and offer treatment in a proper clinical scenario. In addition, in patients with unclassified autoinflammatory syndromes, genetic testing screening only for the hereditary periodic fever syndromes (FMF, TRAPS, CAPS, and MVK) yields few positive results. ${ }^{[15]}$
Therefore, a careful and thorough history taking to look for more typical clinical phenotypes is imperative before ordering the genetic testing. In addition, other gene mutations like NOD2 may be ordered to cover other SAIDs, i.e., NOD2associated disease.

Due to the usage of the more advanced molecular technology, more than one genetic mutation or variant may be detected. If two or more mutations from two or more different genes are detected, it would make the interpretation of the results tricky and difficult. Such terms as digenic, trigenic, and polygenic variants are used in inheritable disorders. More recently, digenic inheritance has been proposed as another pattern of inheritance in SAIDs. True digenic inheritance is when biallelic or even triallelic mutations in two distinct genes, in cis or in trans, are necessary and sufficient to cause pathology with a defined diagnosis. ${ }^{[16]}$ To confirm digenic inherence, a familial separation study is needed. Clinically, two or more gene mutations/variants are identified in an individual patient with SAID, and more commonly, such combinations may be MEFV and NOD2.. ${ }^{[17]}$ These variants may not be truly digenic; instead, they may be synergistic in contribution to variable disease expression in an individual. In the end, a careful correlation between phenotypes and genotypes is helpful in reaching a correct diagnosis.

\section{Conflict of interest}

Qingping Yao is an Editorial Board Member of the journal. The article was subject to the journal's standard procedures, with peer review handled independently of this member and his research groups.

Funding

There is no financial funding support for this study.

\section{References}

[1] Martinez-Quiles N, Goldbach-Mansky R. Updates on Autoinflammatory Diseases. Curr Opin Immunol, 2018;55:97-105. doi: 10.1016/j.coi.2018.09.014.

[2] Yao Q. Nucleotide-Binding Oligomerization Domain Containing 2: Structure, Function, and Diseases. Semin Arthritis Rheum, 2013;43(1):125-130. doi: 10.1016/j.semarthrit.2012.12.005.

[3] Guttmacher AE, Collins FS. Genomic Medicine - A Primer. N Engl J Med, 2002;347(19):1512-1520. doi: 10.1056/NEJMra012240.

[4] Rama M, Mura T, Kone-Paut I, et al. Is Gene Panel Sequencing More Efficient than Clinical-based Gene Sequencing to Diagnose Autoinflammatory Diseases? A Randomized Study. Clin Exp Immunol, 2021;203(1):105-114. doi: 10.1111/cei.13511.

[5] Kosukcu C, Taskiran EZ, Batu ED, et al. Whole Exome Sequencing in Unclassified Autoinflammatory Diseases: More Monogenic Diseases in the Pipeline? Rheumatology (Oxford), 2021;60(2):607-616. doi: 10.1093/rheumatology/keaa165.

[6] Kastner DL, Aksentijevich I, Goldbach-Mansky R. Autoinflamma- tory Disease Reloaded: A Clinical Perspective. Cell, 2010;140(6): 784-790. doi: 10.1016/j.cell.2010.03.002.

[7] Eyal O, Shinar Y, Pras M, et al. Familial Mediterranean Fever: Penetrance of the p.[Met694Val];[Glu148GIn] and p.[Met694Val];[=] Genotypes. Hum Mutat, 2020;41(11):1866-1870. doi: 10.1002/ humu.24090.

[8] Sugiura T, Kawaguchi Y, Fujikawa S, et al. Familial Mediterranean Fever in Three Japanese Patients, and a Comparison of the Frequency of MEFV Gene Mutations in Japanese and Mediterranean Populations. Mod Rheumatol, 2008;18(1):57-59. doi: 10.1007/ s10165-007-0003-2.

[9] Verma D, Lerm M, Julinder RB, et al. Gene Polymorphisms in the NALP3 Inflammasome are Associated with Interleukin-1 Production and Severe Inflammation: Relation to Common Inflammatory Diseases? Arthritis Rheum, 2008;58(3):888-894. doi: 10.1002/art.23286. [10] Naselli A, Penco F, Cantarini L, et al. Clinical Characteristics of Patients Carrying the Q703K Variant of the NLRP3 Gene: A 10-year 
Multicentric National Study. J Rheumatol, 2016;43(6):1093-1100. doi: 10.3899/jrheum.150962.

[11] Kuemmerle-Deschner JB, Verma D, Endres T, et al. Clinical and Molecular Phenotypes of Low-Penetrance Variants of NLRP3: Diagnostic and Therapeutic Challenges. Arthritis Rheumatol, 2017;69(11):2233-2240. doi: 10.1002/art.40208.

[12] Jeru I, Duquesnoy P, Fernandes-Alnemri T, et al. Mutations in NALP12 Cause Hereditary Periodic Fever Syndromes. Proc Natl Acad Sci U S A, 2008;105(5):1614-1619. doi: 10.1073/pnas.0708616105. [13] Vitale A, Rigante D, Lucherini OM, et al. The Role of the F402L Allele in the NLRP12-Autoinflammatory Disorder. Reply to: F402L Variant in NLRP12 in Subjects with Undiagnosed Periodic Fevers and in Healthy Controls, De Pieri et al. Clin Exp Rheumatol, 2014;32(6):994.
[14] Richards S, Aziz N, Bale S, et al. Standards and Guidelines for the Interpretation of Sequence Variants: A Joint Consensus Recommendation of the American College of Medical Genetics and Genomics and the Association for Molecular Pathology. Genet Med, 2015;17(5):405-424. doi: 10.1038/gim.2015.30.

[15] Simon A, van der Meer JWM, Vesely R, et al. Approach to Genetic Analysis in the Diagnosis of Hereditary Autoinflammatory Syndromes. Rheumatology (Oxford), 2006;45(3):269-273. doi: 10.1093/rheumatology/kei138.

[16] Deltas C. Digenic Inheritance and Genetic Modifiers. Clin Genet, 2018;93(3):429-438. doi: 10.1111/cge.13150.

[17] Yao Q, Li E, Shen B. Autoinflammatory Disease with Focus on NOD2-Associated Disease in the Era of Genomic Medicine. Autoimmunity, 2019;52(2):48-56. doi: 10.1080/08916934.2019.1613382. 